\title{
Real-Time Visualization of Solid-Phase Ion Migration Kinetics on Nanowire Monolayer
}

Zhen He, ${ }^{\mathrm{a}, \dagger}$ Li-Ge Chang, ${ }^{\mathrm{b}, \dagger}$ Yue Lin,${ }^{\mathrm{c}, \dagger}$ Feng-Lei Shi, ${ }^{\mathrm{d}}$ Ze-Dong Li, ${ }^{\mathrm{c}}$ Jin-Long Wang, ${ }^{\mathrm{a}}$ Yi Li, ${ }^{\text {a }}$ Rui Wang, ${ }^{\mathrm{a}}$ Qing-Xia Chen, ${ }^{\mathrm{a}}$ Yu-Yang Lu, ${ }^{\mathrm{b}}$ Qing-Hua Zhang, ${ }^{\mathrm{e}}$ Lin Gu, ${ }^{\mathrm{e}}$ Yong $\mathrm{Ni},{ }^{\text {b Jian-Wei Liu }}{ }^{*, a}$, Jian-Bo $\mathrm{Wu}^{*, \mathrm{~d}}$, and Shu-Hong $\mathrm{Yu}^{*, \mathrm{a}}$

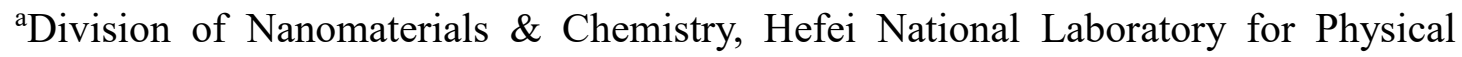
Sciences at the Microscale, Institute of Energy, Hefei Comprehensive National Science Center, CAS Center for Excellence in Nanoscience, Collaborative Innovation Center of Suzhou Nano Science and Technology, Department of Chemistry, University of Science and Technology of China, Hefei 230026, China. ${ }^{b}$ CAS Key Laboratory of Mechanical Behavior and Design of Materials, Department of Modern Mechanics, University of Science and Technology of China, Hefei, Anhui, 230027, China. ${ }^{\mathrm{c} H e f e i}$ National Laboratory for Physical Sciences at Microscale, University of Science and Technology of China, Hefei 230026, China. 'State Key Laboratory of Metal Matrix Composites, School of Materials Science and Engineering, Center of Hydrogen Science, Shanghai

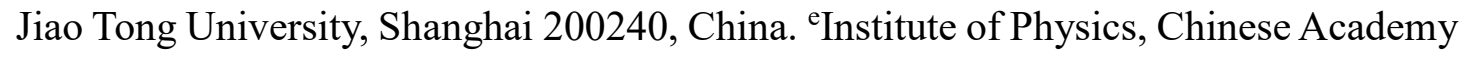
of Sciences, Beijing 100190, China. 


\section{Contents}

\section{Supplementary Notes}

Note S1. Derivation of concentration evolution equation.

Note S2. Calculation of strain and stress.

Note S3. Material parameters.

\section{Supplementary Figures}

Figure S1. The proportion of Ag distribution after migration.

Figure S2. The crystal structure of reacted Te nanowires after the Ag migration.

Figure S3. TEM image of the electron beam induced reaction on the special designed Ag-Te co-assembly sample.

Figure S4. Ag nanowire before and after the electron beam irradiation.

Figure S5. Te nanowire before and after the electron beam irradiation.

Figure S6. STEM image of the emerged heterostructure in the edge of reaction zone.

Figure S7. Structure evolution of Ag atoms invading Te nanostructure from ab initio simulation.

Figure S8. Comparison of the Ag invading process with and without the surface diffusion at the same time simulated by our phase-field model.

Figure S9. The atom distribution in axial direction of nanowire.

Figure S10. The hydrostatic stress evolution considering the surface diffusion time.

Figure S11. The connection "bridge" phenomenon in the reaction zone.

Figure S12. (a-d) EDS line scan of Se, Te and Ag at the reaction zone after migration. Figure $\mathrm{S} 13$. The migration of $\mathrm{Cu}$ on $\mathrm{Te} \mathrm{NWs}$ under electron beam irradiation.

\section{Supporting Experimental Section}

\section{Supplementary Notes}

\section{Derivation of concentration evolution equation.}

We adopt the smoothed boundary method to combine the phase transformation in the bulk Te NW with fast diffusion of Ag atom along the Te NW surface. A domain parameter $\varphi$ is introduced to distinguish the bulk nanowire, the surrounding vacuum and surface zone of the nanowire, where $\varphi$ equals 1 in the bulk of the NW and 0 in the vacuum, varying smoothly from 0 to 1 within the surface zone. Cahn-Hilliard equation is used to describe the phase transformation in the bulk of the nanowire. ${ }^{1}$

$$
\frac{\partial \hat{c}}{\partial t}=\frac{D_{b}}{\rho k_{B} T} \nabla^{2} \mu \quad \varphi=1
$$


and the concentration evolution for the surface zone observes the conservation law, ${ }^{2}$

$$
\frac{\partial \hat{c}}{\partial t}=\frac{D_{s}}{\rho k_{B} T} \nabla_{s}^{2} \mu-\frac{1}{\rho \lambda}\left(J_{\text {int }}-J_{\text {appl }}\right) \quad 0<\varphi<1
$$

Here, $\rho$ is the number of Ag atom per unit volume. $k_{B}$ and $T$ are Boltzmann's constant and the absolute temperature, respectively. $D_{b}$ and $D_{s}$ represent the diffusion coefficient in the bulk and surface, respectively. $\lambda$ is the characteristic thickness of the surface zone. $\nabla_{s}$ is the surface gradient operator. $\mu$ is the chemical potential, which is defined as $\mu=\delta E / \delta \hat{c} . E$ is the total energy of the system as shown in Eq.(2). $J_{\text {int }}$ denotes the flux from the surface into the bulk, $J_{a p p l}$ is the applied flux imposed at the left side of the nanowire as shown in Figure S2.

To effectively simulate the surface diffusion and phase transformation in the bulk nanowire, we adopt the smoothed boundary method via multiplying the domain parameter $\varphi$ on the both sides of Eq. (S1),

$$
\varphi \frac{\partial \hat{c}}{\partial t}=\frac{D_{b}}{\rho k_{B} T} \nabla \cdot(\varphi \nabla \mu)-\frac{D_{b}}{\rho k_{B} T} \nabla \varphi \cdot \nabla \mu
$$

Recalling the relationship between the normal vector $\mathbf{n}$ (pointing to the nanowire) and the domain parameter, i.e. $\mathbf{n}=\nabla \varphi /|\nabla \varphi|$, and the flux $\mathbf{J}_{\text {int }}=-D_{b} \nabla \mu /\left(k_{B} T\right)$, we can obtain $^{2}$

$$
\frac{\partial \hat{c}}{\partial t}=\frac{D_{b}}{\rho \varphi k_{B} T} \nabla \cdot(\varphi \nabla \mu)+\frac{1}{\rho \varphi}|\nabla \varphi| J_{\mathrm{int}}
$$

Then, we can combine the surface diffusion equation (Eq. (S2)) with the Cahn Hilliard equation (Eq. (S4)) by substituting the term $J_{\text {int, }}$

$$
\frac{\partial \hat{c}}{\partial t}=\frac{D_{b}}{\varphi \rho k_{B} T} \nabla \cdot(\varphi \nabla \mu)+\frac{|\nabla \varphi|}{\rho \varphi}\left(\nabla_{s} \cdot \frac{\lambda D_{s}}{k_{B} T} \nabla_{s} \mu+J_{a p p l}-\rho \lambda \frac{\partial \hat{c}}{\partial t}\right) .
$$

Finally, it can be written in a simpler form as Eq. (1).

\section{Calculation of strain and stress.}

We adopt the phase field microelasticity theory to obtain the distribution of strain and stress. The elastic strain can be expressed as 


$$
\varepsilon_{i j}^{e}(\mathbf{r})=\varepsilon_{i j}(\mathbf{r})-\varepsilon_{i j}^{c}(\mathbf{r})
$$

where $\varepsilon_{i j}^{c}(\mathbf{r})$ is the chemical eigenstrain tensor that can be given by $\varepsilon_{i j}^{c}(\mathbf{r})=\beta\left(\hat{c}-c_{0}\right) \delta_{i j}, \beta$ is the expansion coefficient, $c_{0}$ is the reference concentration and $\delta_{i j}$ is Kronecker delta function. The total strain $\varepsilon_{i j}(\mathbf{r})$ can be written as. ${ }^{3,4}$

$$
\varepsilon_{i j}(\mathbf{r})=\bar{\varepsilon}_{i j}+\frac{1}{2} \int_{|\mathbf{k}| \neq 0}\left[\tilde{n}_{i} \Omega_{j k}(\tilde{\mathbf{n}})+\tilde{n}_{j} \Omega_{i k}(\tilde{\mathbf{n}})\right]\left\{C_{k l m n}^{0} \varepsilon_{m n}^{0}(\mathbf{r})\right\}_{\mathbf{k}} \tilde{n}_{l} e^{i \mathbf{k} \cdot \mathbf{r}} \frac{d^{3} k}{(2 \pi)^{3}}
$$

where the average strain $\bar{\varepsilon}_{i j}$ is given by $\bar{\varepsilon}_{i j}=\int_{V} \varepsilon_{i j}(\mathbf{r}) d^{3} r / V . \tilde{n}_{i}$ is a component of the unit vector $\tilde{\mathbf{n}}=\mathbf{k} /|\mathbf{k}|$ in the Fourier space, where $\mathbf{k}$ is the wave vector and $|\mathbf{k}|$ is its modulus. $\Omega_{i j}(\tilde{\mathbf{n}})$ is the Green function tensor. $\left\{C_{k l m n}^{0} \varepsilon_{m n}^{0}(\mathbf{r})\right\}_{\mathbf{k}}$ denotes the Fourier transformation of $C_{k l m n}^{0} \varepsilon_{m n}^{0}(\mathbf{r}) \cdot \varepsilon_{i j}^{0}(\mathbf{r})$ is defined as the virtual eigenstrain when introducing a elastically and structurally inhomogeneous computational system, and it is determined by a time-dependent Ginzburg-Landau type equation. ${ }^{3}$

$$
\frac{\partial \varepsilon_{i j}^{0}(\mathbf{r}, t)}{\partial t}=-L_{i j k l} \frac{\delta E^{\mathrm{inhom}}}{\delta \varepsilon_{k l}^{0}(\mathbf{r}, t)}
$$

where $L_{i j k l}$ is the kinetic coefficient. $E^{\text {inhom }}$ is elastic energy of the elastically and structurally inhomogeneous system energy which can be obtained from

$$
\begin{aligned}
E^{\mathrm{inhom}}= & \frac{1}{2} \int_{V}\left[C_{i j m n}^{0} \Delta C_{m n p q}^{-1}(\mathbf{r}) C_{p q k l}^{0}-C_{i j k l}^{0}\right]\left[\varepsilon_{i j}^{0}(\mathbf{r})-\varepsilon_{i j}^{c}(\mathbf{r})\right]\left[\varepsilon_{k l}^{0}(\mathbf{r})-\varepsilon_{k l}^{c}(\mathbf{r})\right] d V \\
& +\frac{1}{2} \int_{V} C_{i j k l}^{0} \varepsilon_{i j}^{0}(\mathbf{r}) \varepsilon_{k l}^{0}(\mathbf{r}) d V+\frac{V}{2} C_{i j k l}^{0} \bar{\varepsilon}_{i j} \bar{\varepsilon}_{k l}-\bar{\varepsilon}_{i j} \int_{V} C_{i j k l}^{0} \varepsilon_{k l}^{0}(\mathbf{r}) d V \\
& -\frac{1}{2} \int_{|\mathbf{k}| \neq 0} \tilde{n}_{i} \tilde{\sigma}_{i j}^{0}(\mathbf{k}) \Omega_{j k}(\tilde{\mathbf{n}}) \tilde{\sigma}_{k l}^{0}(\mathbf{k})^{\prime} \tilde{n}_{l} \frac{d^{3} k}{(2 \pi)^{3}},
\end{aligned}
$$

where $\sigma_{i j}^{0}(\mathbf{r})$ is the eigenstrains that obeys Hooke's law $\sigma_{i j}^{0}(\mathbf{r})=C_{i j k l}^{0} \varepsilon_{i j}^{0}(\mathbf{r}) \cdot \tilde{\sigma}_{i j}^{0}(\mathbf{k})$ is the Fourier transform of $\sigma_{i j}^{0}(\mathbf{r})$ and $\tilde{\sigma}_{k l}^{0}(\mathbf{k})^{\prime}$ is complex conjugate of $\tilde{\sigma}_{k l}^{0}(\mathbf{k})$. $\Delta C_{m n p q}^{-1}$ is the inverse of $\Delta C_{m n p q}$. The relationship between $\Delta C_{i j k l}(\mathbf{r})$ and $C_{i j k l}^{0}$ is $\Delta C_{i j k l}(\mathbf{r})=C_{i j k l}^{0}-C_{i j k l}(\mathbf{r})$. Here, the reference modulus $C_{i j k l}^{0}$ is set as modulus of the nanowire. As a result, $\Delta C_{i j k l}(\mathbf{r})=0$ in the nanowire and $\Delta C_{i j k l}(\mathbf{r})=C_{i j k l}^{0}$ in the 
surrounding vacuum space. Once the total strain is obtained by Eq. (S7), the elastic stress will be identified according to Hooke's law,

$$
\sigma_{i j}(\mathbf{r})=C_{i j k l}(\mathbf{r})\left[\varepsilon_{k l}(\mathbf{r})-\varepsilon_{k l}^{c}(\mathbf{r})\right]
$$

\section{Material parameters.}

\section{Supplementary Table 1}

\begin{tabular}{cll}
\hline symbol & parameter & value \\
\hline$\rho$ & number of atoms per unit volume & $2.82 \times 10^{28} \mathrm{~m}^{-3 \mathrm{a}}$ \\
$\kappa$ & gradient coefficient & $4.5 \times 10^{-11} \mathrm{~J} \cdot \mathrm{m}^{-1 \mathrm{~b}}$ \\
$\lambda$ & characteristic thickness of the surface & $1 \mathrm{~nm}^{\mathrm{c}}$ \\
$v$ & Poisson's ratio & $0.3^{\mathrm{a}}$ \\
$G$ & shear modulus of Te & $16 \mathrm{GPa}^{\mathrm{a}}$ \\
$\omega$ & potential height & $5.76 \times 10^{7} \mathrm{~J} \mathrm{~m}^{-3}$ \\
$k_{\mathrm{B}}$ & Boltzmann constant & $1.38 \times 10^{-23} \mathrm{JK}^{-1}$ \\
$T$ & temperature & $300 \mathrm{~K}$ \\
$\beta$ & expansion coefficient & $0.256^{\mathrm{b}}$ \\
$c_{\text {max }}$ & maximum concentration & $4.68 \times 10^{4} \mathrm{molm}^{-3} \mathrm{a}$ \\
$l$ & characteristic length & $20 \mathrm{~nm}$ \\
$D_{s}$ & surface diffusion coefficient & $2.79 \times 10^{-11} \mathrm{~cm}^{2} / \mathrm{s}$ \\
$D_{b}$ & bulk diffusion coefficient & $1.25 \times 10^{-13} \mathrm{~cm}^{2} / \mathrm{s}$ \\
& &
\end{tabular}

${ }^{a}$ obtained from the website: https://materialsproject.org

${ }^{\mathrm{b}}$ calculated as follows

${ }^{\mathrm{c}}$ measured from the experiment

The determination of expansion coefficient $\beta$ : According to the measured $33 \%$ increase in the diameter of the $\mathrm{Ag}_{2} \mathrm{Te} \mathrm{NW}$ compared to the intact $\mathrm{Te} \mathrm{NW}$, the volumetric strain $\theta=\left(d V-d V_{0}\right) / d V_{0}$, is calculated as 0.769 . It is assumed that the chemical eigenstrains is isotropic, i.e. $\varepsilon_{11}^{c}=\varepsilon_{22}^{c}=\varepsilon_{33}^{c}$, and recalling $\varepsilon_{i j}^{c}(\mathbf{r})=\beta\left(\hat{c}-c_{0}\right) \delta_{i j}$, where the reference concentration is set as zero. Consequently, the expansion coefficient $\beta$ has the value 0.256 .

The gradient coefficient $\kappa$ can be determined by the following equations, ${ }^{4}$ $\kappa=l_{\text {int }}^{2} \omega / 32$ and $\gamma=\sqrt{k \omega / 2} / 2$. Here, $\gamma$ is the interfacial free energy, which is 
estimated as $0.5 \mathrm{~J} / \mathrm{m}^{2} . l_{\text {int }}$ is the interface width between the two phases, which is estimated about $1 \mathrm{~nm}$ according to Figure 3. Thus, we can calculate $\kappa$ and $w$ as $3.5 \times 10^{-}$ ${ }^{10} \mathrm{~J} \mathrm{~m}^{-1}$ and $5.7 \times 10^{9} \mathrm{~J} \mathrm{~m}^{-3}$, respectively.

The calculation of bulk diffusion coefficient is based on the relationship between Ag atom flux $(\mathrm{J})$ and the concentration gradient: $J=q /\left(\pi R^{2} t\right)=-D_{b} d C_{A_{g}} / d x$. Here, q is the molar quantity of $\mathrm{Ag}$ which equals to $\mathrm{C}_{0} \times \mathrm{V}_{\mathrm{Ag}}$. $\mathrm{t}$ is the measured time which is set as $15 \mathrm{~s}$, and $\mathrm{dC}_{\mathrm{Ag}} / \mathrm{dx}$ is the corresponding concentration gradients of $\mathrm{Ag}$ according to Supplementary Fig. 1 and $\mathrm{dC}_{\mathrm{Ag}} / \mathrm{dx}$ is calculated as $0.7813 \times \mathrm{C}_{0}$. $\mathrm{R}$ is the radius of the nanowire, which is measured as $3.5 \mathrm{~nm}$. Finally, the bulk diffusion coefficient was estimated to be $1.25 \times 10^{-13} \mathrm{~cm}^{2} \mathrm{~s}^{-1}$. It should be pointed out that the surface diffusion coefficient is difficult to calculate so that its value is estimated as $2.79 \times 10^{-11} \mathrm{~cm}^{2} \mathrm{~s}^{-1}$ in our simulation. 


\section{Supplementary Figures}

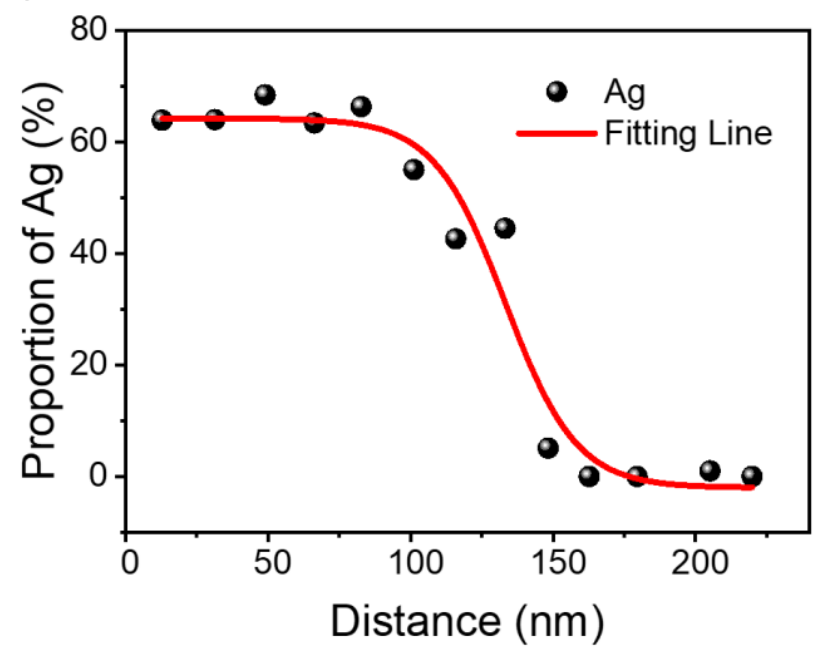

Figure S1.The proportion of Ag calculated from Fig. 1d.
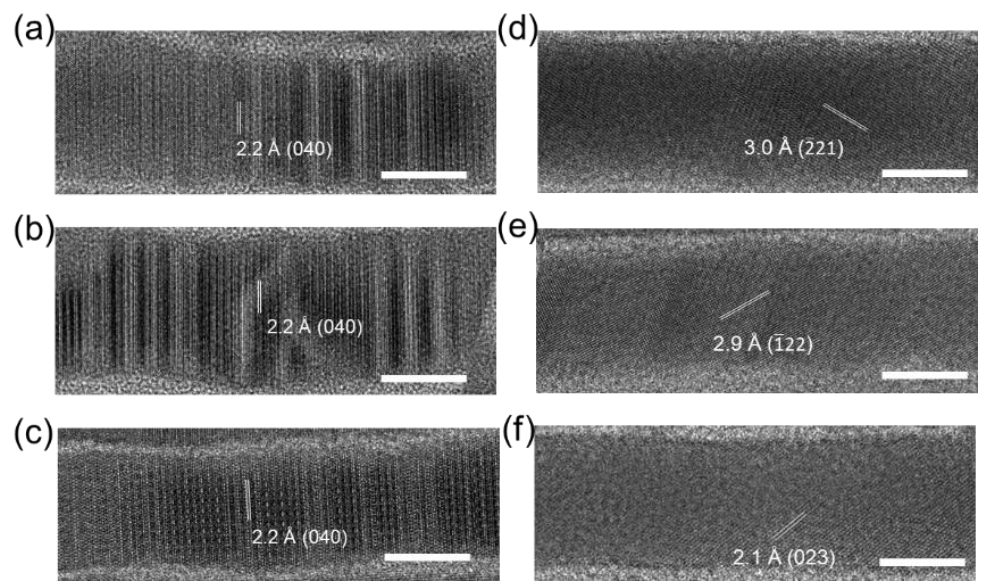

Figure S2. The crystal structure of reacted Te nanowires after the Ag migration. (a-f) HRTEM images of the reacted Te nanowire from the $1^{\text {st }}$ to $6^{\text {th }}$ nanowires. The scale bars are $5 \mathrm{~nm}$.

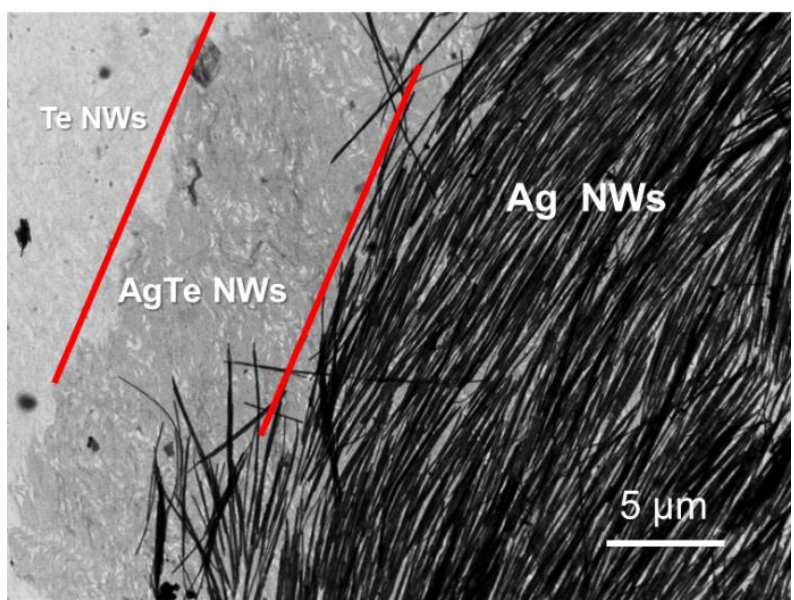

Figure S3. TEM image of the electron beam induced reaction on the special designed Ag-Te coassembly sample (left panel are Te nanowires and right panel are Ag nanowires). The average reaction length can reach as long as $10 \mu \mathrm{m}$. 

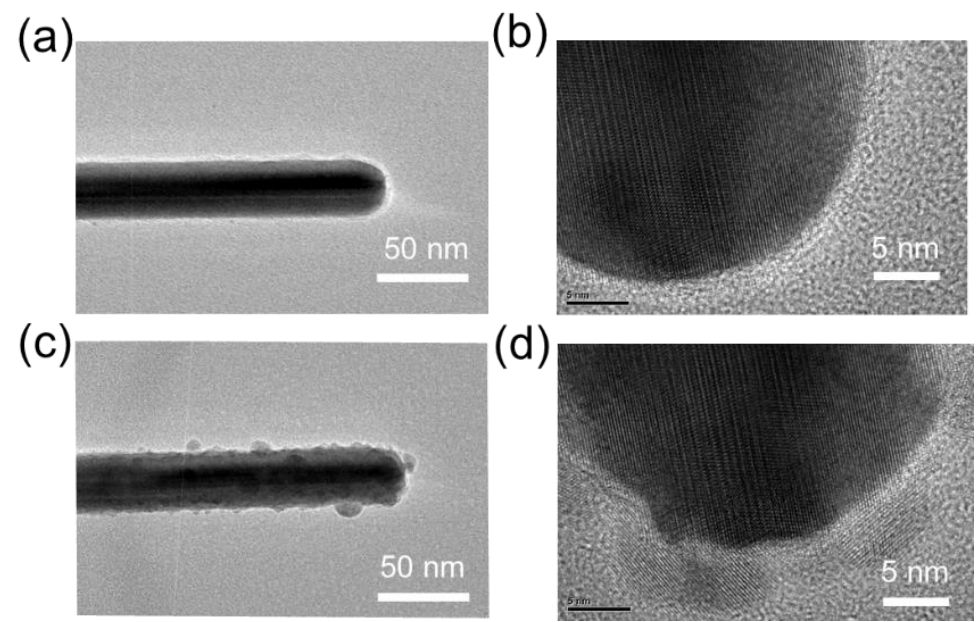

Figure S4. Ag nanowire before and after the electron beam irradiation. (a-b) TEM and the corresponding HRTEM images of Ag nanowire with uniform morphology before the electron beam irradiation; (c-d) TEM and the corresponding HRTEM images of Ag nanowire with rough morphology after the electron beam irradiation.

(a)

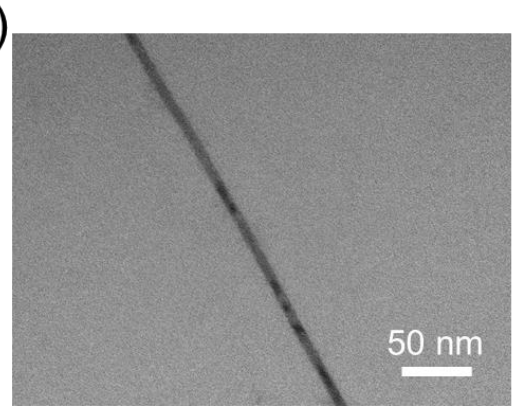

(b)

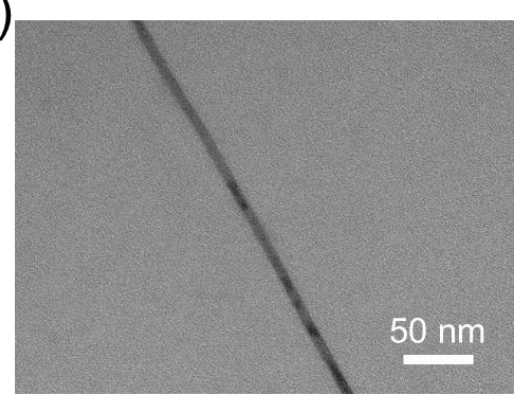

Figure S5. Te nanowire before and after the electron beam irradiation. (a) TEM image of Te nanowire before the electron beam irradiation; (b) TEM image of Te nanowire after the electron beam irradiation, showing uniform morphology.

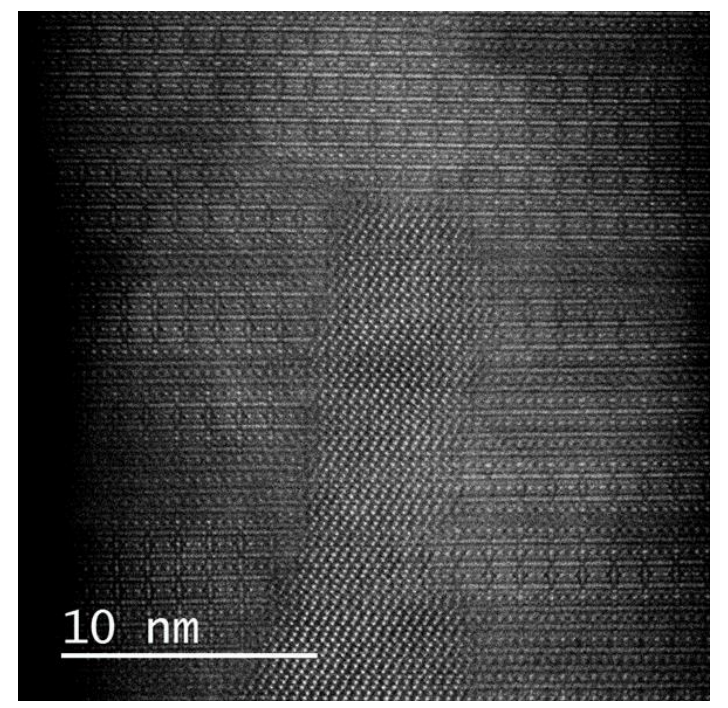

Figure S6. STEM image of the emerged heterostructure in the edge of reaction zone. The edge of core-shell structure. 


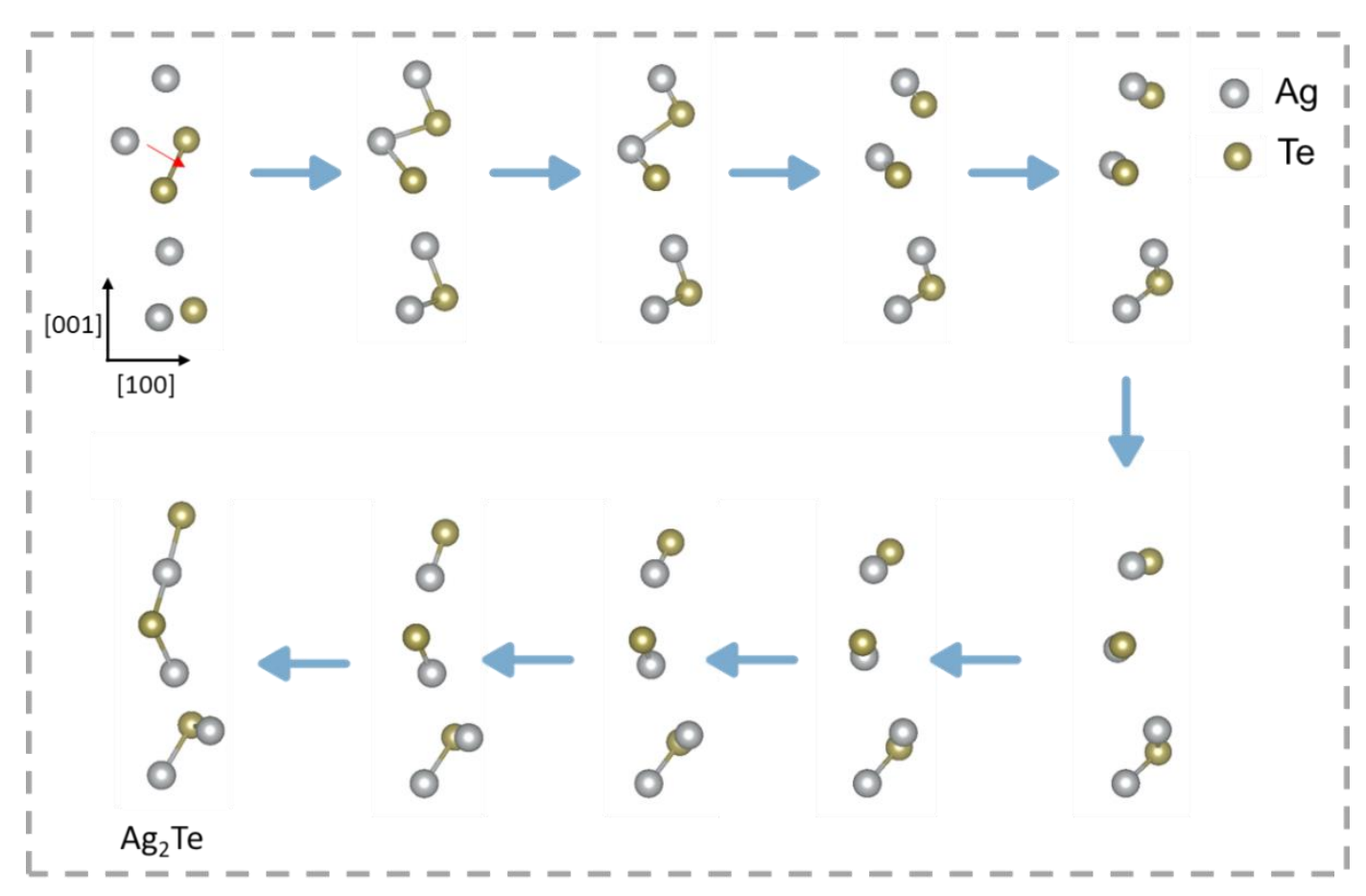

Figure S7. Structure evolution of Ag atoms invading Te nanostructure from ab initio simulation. $\mathrm{Ag}$ atoms invade into the (101) facet of Te.

(a)
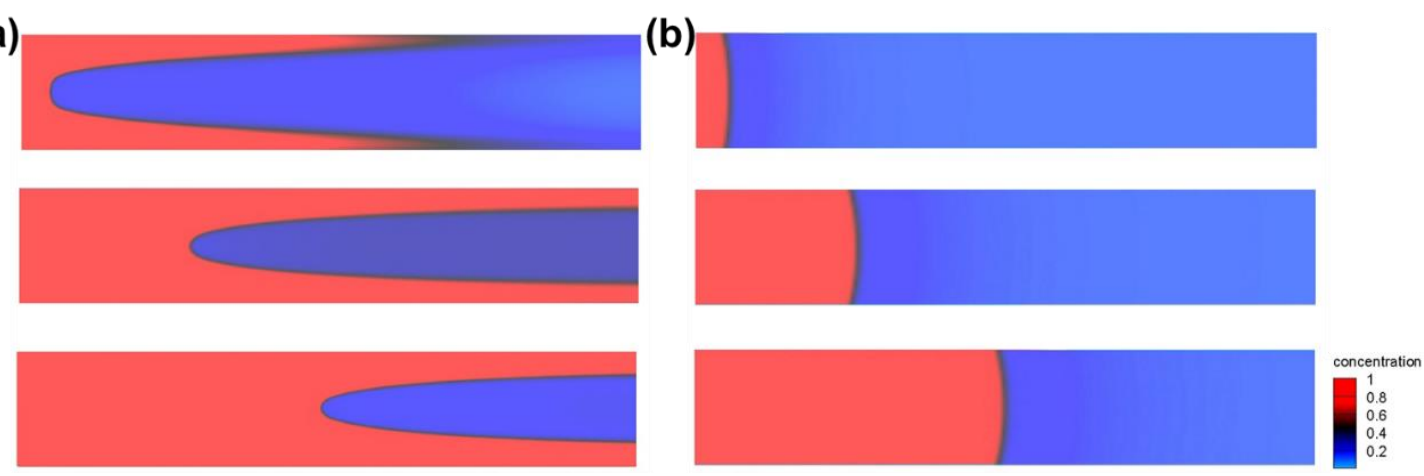

Figure S8. Comparison of the Ag invading process with and without the surface diffusion at the same time simulated by our phase-field model. (a) Snapshots of Ag invading process considering the surface diffusion at time $120 \mathrm{~s}, 230 \mathrm{~s}$ and $340 \mathrm{~s}$. (b) Snapshots of Ag invading process neglecting the surface diffusion at the same times. 


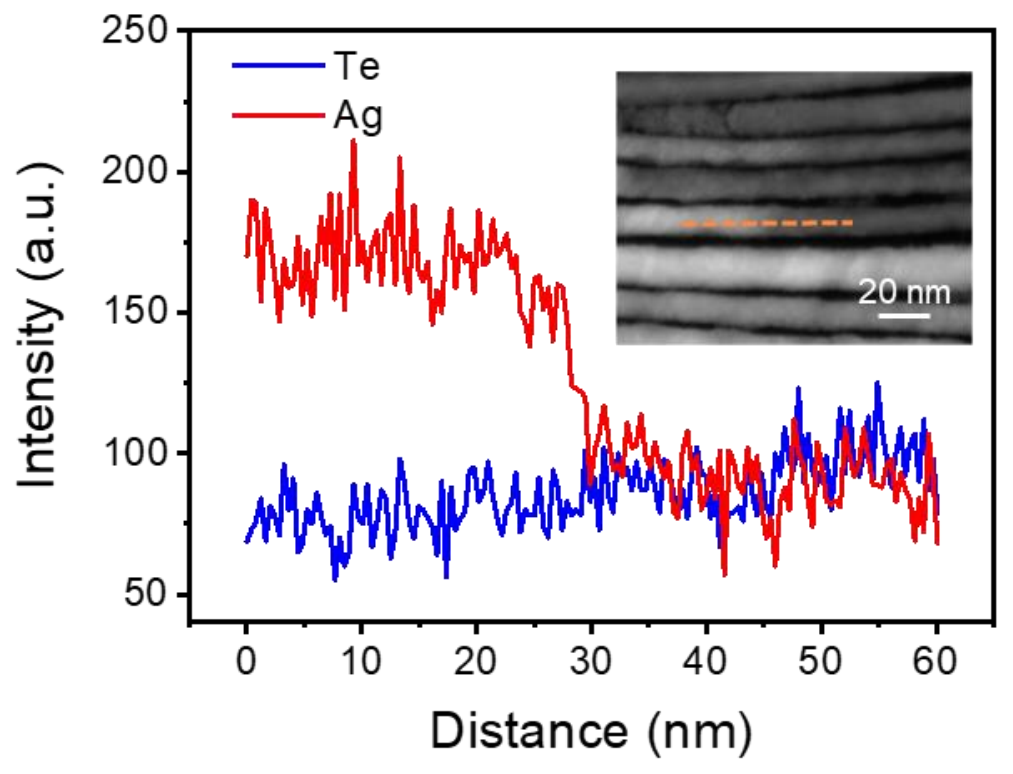

Figure S9. The atom distribution in axial direction of nanowire. The inset was the HRTEM image of the reaction zone.

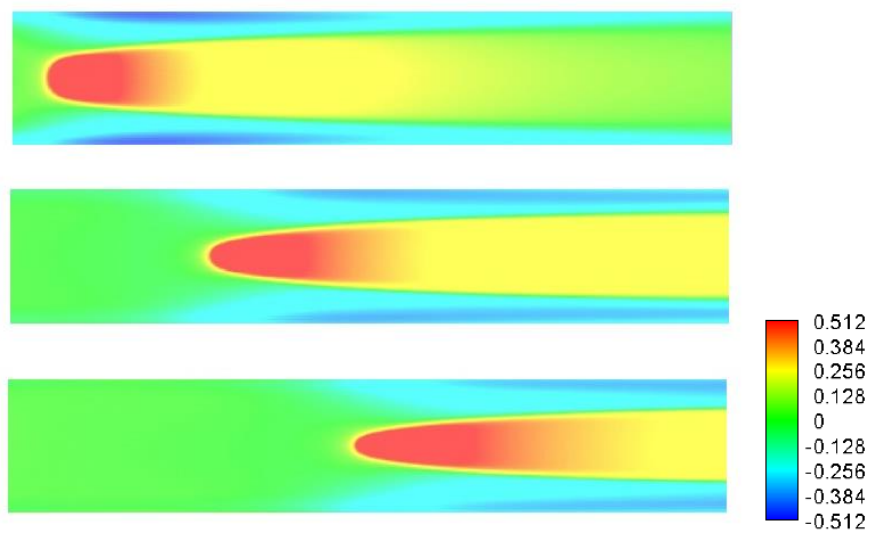

Figure S10. The hydrostatic stress evolution considering the surface diffusion at time $120 \mathrm{~s}, 230 \mathrm{~s}$ and $340 \mathrm{~s}$, corresponding to the concentration distribution in Figure S8a. The color bar indicates the level of hydrostatic stress with the unit: GPa. 
(a)

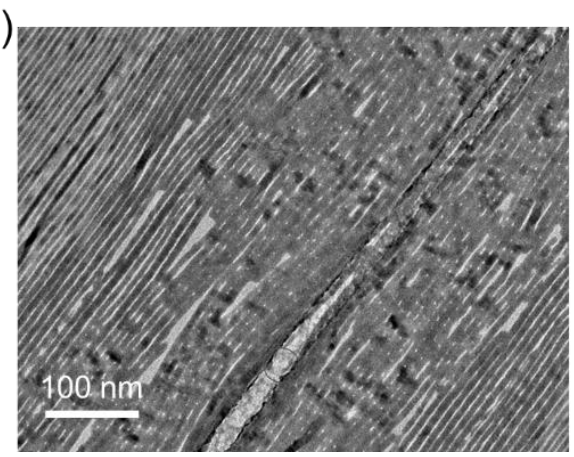

(b)

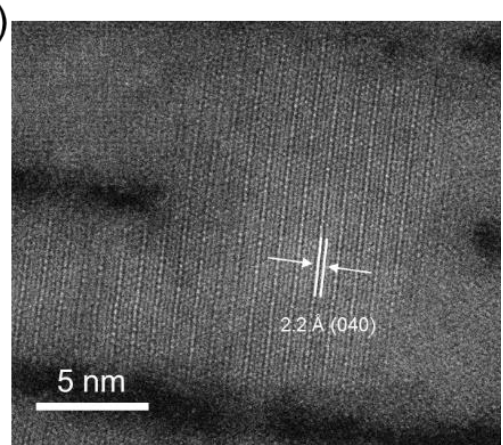

Figure S11. The connection "bridge" phenomenon in the reaction zone. (a) TEM image of the reaction zone after completely migration; (b) High-resolution STEM image of connection "bridge" between telluride nanowires and the two nanowires grow into an intact crystal.

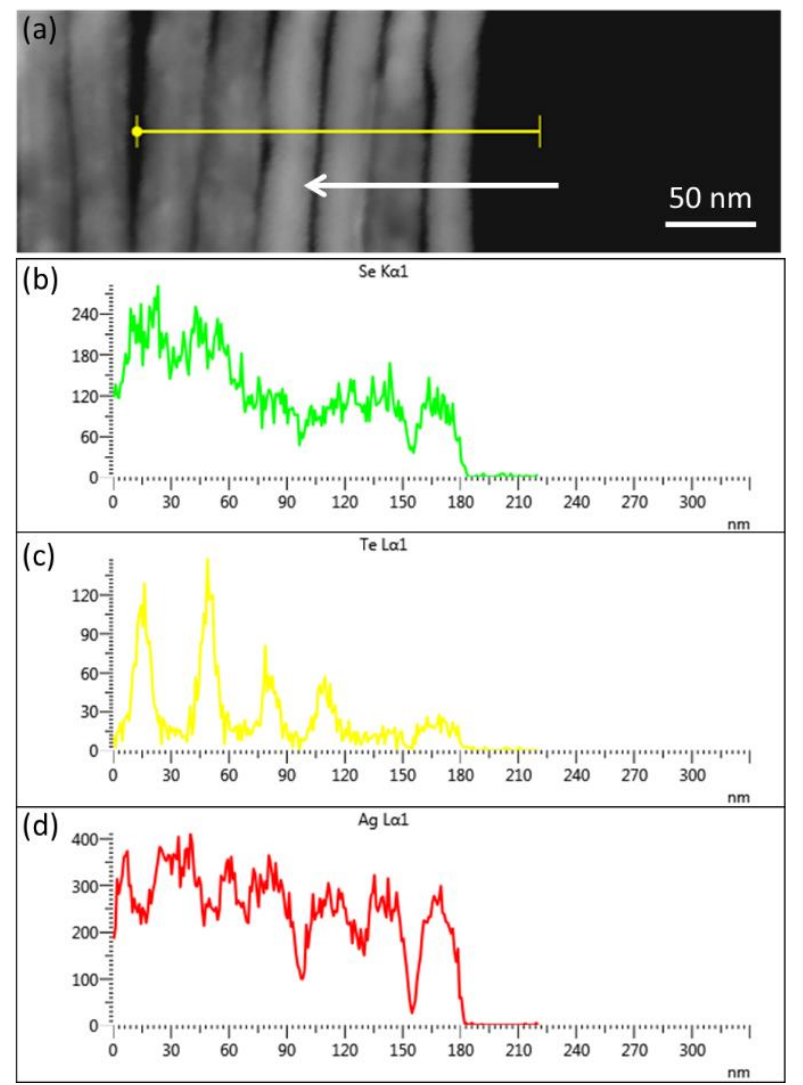

Figure S12. (a-d) EDS line scan of Se, Te and $\mathrm{Ag}$ at the reaction zone after migration. 
(a)

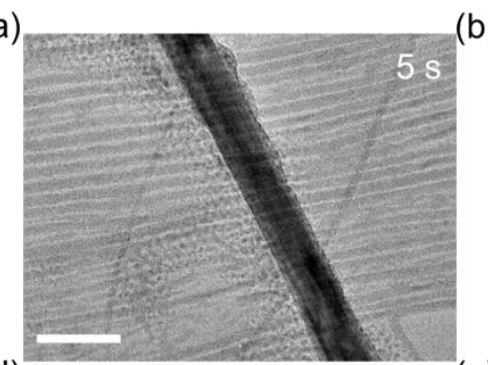

(d)

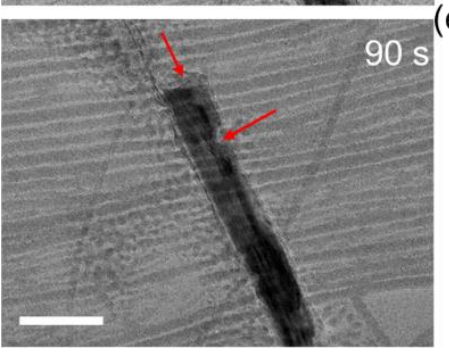

(b)
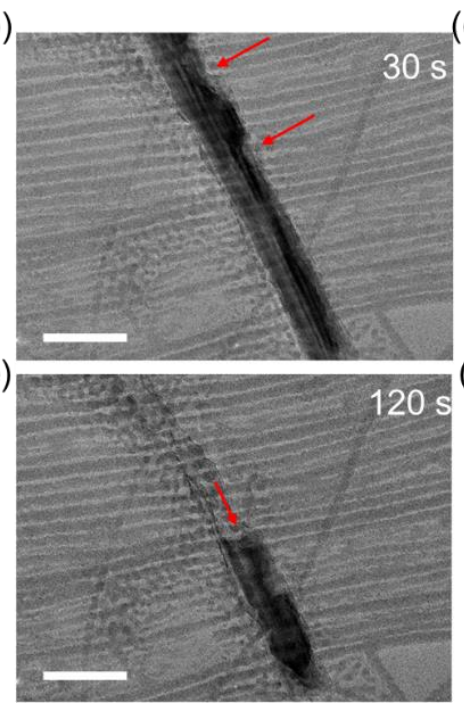
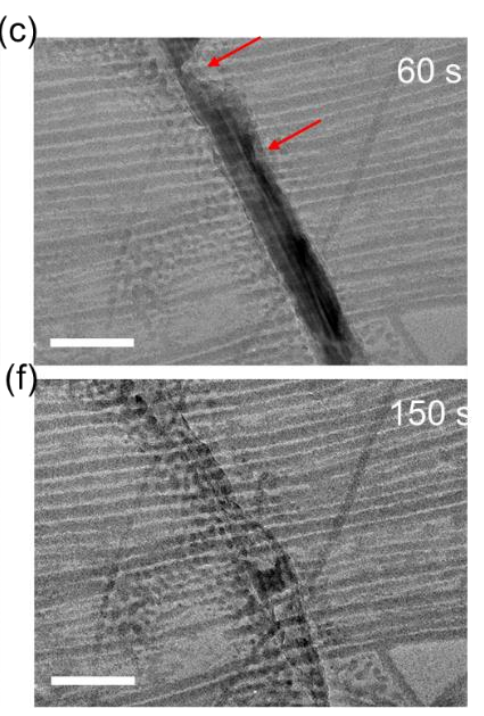

Figure S13. The migration of $\mathrm{Cu}$ on Te NWs under electron beam irradiation. (a-f) Time sequential TEM images of $\mathrm{Cu}$ migration process showing the $\mathrm{Cu} \mathrm{NW}$ was gradually disappeared. The scale bars in (a-f) are $50 \mathrm{~nm}$.

\section{References}

1. Singh, G. K.; Ceder, G.; Bazant, M. Z., Intercalation dynamics in rechargeable battery materials: General theory and phase-transformation waves in $\mathrm{LiFePO}_{4}$. Electrochim. Acta 2008, 53 (26), 7599-7613. 2. Yu, H.-C.; Choe, M.-J.; Amatucci, G. G.; Chiang, Y.-M.; Thornton, K., Smoothed Boundary Method for simulating bulk and grain boundary transport in complex polycrystalline microstructures. Computational Materials Science 2016, 121, 14-22.

3. Wang, Y. U.; Jin, Y. M.; Khachaturyan, A. G., Phase field microelasticity theory and modeling of elastically and structurally inhomogeneous solid. Journal of Applied Physics 2002, 92 (3), 1351.

4. Cahn, J. W.; Hilliard, J. E., Free Energy of a Nonuniform System. I. Interfacial Free Energy. The Journal of Chemical Physics 1958, 28 (2), 258. 